3. Jianming Jin, The Finite Element Method in Electromagnetics, John Wiley \& Sons, New York, 1993.

4. J. Brian Davies. "Finite Element Analysis Waveguides and Cavities," IEEE Trans. Magn., Vol. MAG-29, No. 2, March 1993, pp. 1578-1583.

5. Jin-Fa Lee, Din-Kow Sun, and Zoltan J. Cendes. "Full-Wave Analysis of Dielectric Waveguides Using Tangential Vector Finite Elements," IEEE Trans. Microwave Theory Tech., Vol. MTT39, No. 8, Aug. 1991, pp. 1262-1271.

6. K. Hayata, M. Koshiba, M. Eguchi, and M. Suzuki. "Vectorial Finite-Element Method without any Spurious Solutions for Dielectric Waveguiding Problems Using Transverse Magnetic-Field Component," IEEE Trans. Microwave Theory Tech., Vol. MTT-34, No. 11 , Nov. 1986, pp. 1120-1124.

7. Jin-Fa Lee, "Finite Element Analysis of Lossy Dielectric Waveguides," IEEE Trans. Microwave Theory Tech., Vol. MTT-42, No. 6, June 1994, pp. 1025-1031.

8. R. E. Collin, Field Theory of Guided Waves, IEEE Press, New York, 1991.

9. Yilong Lu and F. Anibal Fernandez, "An Efficient Finite Element Solution of Inhomogeneous Anisotropic and Lossy Dielectric Waveguides," IEEE Trans. Microwave Theory Tech., Vol. MTT-41, No. 6/7, June/July 1993, pp. 1215-1223.

10. Srba R. Cvetkovic, An Ping Zhao, and Minaz Punjani, "An Implementation of the Vectorial Finite Element Analysis of Anisotropic Waveguides through a General-Purpose PDE Software," IEEE Trans. Microwave Theory Tech., Vol. MTT-42, Aug. 1994, pp. 1499-1505.

11. K. Yasuura, K. Shimohara, and T. Miyamoto. "Numerical Analysis of a Thin-Film Waveguide by Mode-Matching Method," $J$. Opt. Soc. Am., Vol. 70, No. 2, Feb. 1980, pp. 1025-1031.

12. M. Koshiba, K. Hayata, and M. Suzuki. "Approximate Scalar Finite-Element Analysis of Anisotropic Optical Waveguides," Electron. Lett., Vol. 18, No. 10, May 1982, pp. 411-413.

Received 1-4-95

Microwave and Optical Technology Letters, 9/3, 156-162

(c) 1995 John Wiley \& Sons, Inc.

CCC 0895-2477/95

\section{I.F. FILTER BANDWIDTH OPTIMIZATION OF COHERENT HETERODYNE ANALOG FM OPTICAL RECEIVER}

\author{
Jingshown Wu and Hen-Wai Tsao \\ Department of Electrical Engineering \\ National Taiwan University \\ Taipei, Taiwan \\ Yang-Han Lee \\ Department of Electrical Engineering \\ Tamkang University \\ Tamsui, Taipei Hsien, Taiwan 25317
}

Keang-Po Ho and Le Pond Chin

Department of Electrical Engineering

National Taiwan University

Taipei, Taiwan

\section{KEY TERMS}

Optical receiver, optical communications, coherent optics

\section{ABSTRACT}

The performance of the coherent heterodyne optical FM receiver employing a discriminator has been simulated under different i.f. filter band- widths to find the optimal bandwidth. The value of the signal-to-noise ratio has been obtained under different values of i.f. filter bandwidth, modulation index, and signal power. The phase noise linewidth is fixed at $\Delta v=10 \mathrm{kHz}$. Even for very small linewidth, the performance of the coherent optical FM receiver is mainly dominated by phase noise. The advantage of FM modulation exists only when received signal power is small. () 1995 John Wiley \& Sons, Inc.

\section{INTRODUCTION}

In coherent heterodyne optical FM communication $[1,2]$, a high modulation index must be employed to improve the SNR of the system. Therefore the bandwidth of the i.f. filter must be increased according to the Carson rule [3]:

$$
B_{\text {i.f. }} \approx 2(\boldsymbol{\beta}+1) f_{m},
$$

where $B_{\text {i.f. }}$ is the bandwidth of the i.f. filter, $\beta$ is the modulation index, and $f_{m}$ is the maximum message frequency. The presence of phase noise means that a larger bandwidth is required. It is important to show how large the bandwidth must be. If the bandwidth is rather wide, more shot noise and thermal noise will be included. Because the coherent optical FM receiver is dominated by phase noise [4], shot noise or thermal noise will not affect the performance even for small signal power, say $-50 \mathrm{dBm}$.

In this article Section 2 describes the simulation model, and Section 3 presents the numerical results and discussion.

\section{SYSTEM DESCRIPTION AND MODELING}

The schematic block diagram of the heterodyne optical receiver is shown in Figure 1. The received electric current of the $p-i-n$ photodiode can be described as

$$
\begin{aligned}
i(t) & =\operatorname{Re}\left\{2 R \sqrt{P_{S} P_{\mathrm{LO}}} \exp ^{j\left[2 \pi f_{\mathrm{i}, \mathrm{f}} t+\phi_{m}(t)+\phi_{n}(t)\right]}\right\}, \\
\phi_{m}(t) & =2 \pi f_{d} \int_{-\infty}^{t} m(t) d t
\end{aligned}
$$

with $R$ being the responsivity $(0.84), P_{\text {LO }}$ the LO laser power, $P_{S}$ the received signal power, $f_{\text {i.f. }}$ the optical intermediate frequency, $\phi_{m}(t)$ the FM signal, $\phi_{n}(t)$ the phase noise, $n_{s}(t)$ the shot noise, $f_{d}$ the peak frequency deviation, and $m(t)$ the message signal. In our simulation, we use the schematic diagram as shown in Figure 2. The current of the $p-i-n$ photodiode is generated according to Eq. (2). To do single-tone simulation only, the message is set to $\phi_{m}(t)=$ $2 \pi f_{d} \sin \left(2 \pi f_{m} t\right)$; there $f_{m}$ is the largest frequency of the band-limited signal. The added noise is generated.

We consider shot noise but no thermal noise. Shot noise is described as

$$
\sigma_{\mathrm{sh}}^{2}=2 e R P_{\mathrm{LO}} B_{\mathrm{iff}}
$$

with $e$ being the electric charge and $B_{\text {i.f. }}$ the bandwidth of the i.f. filter.

The total phase noise $\phi_{n}(t)$ has power spectral density [5]

$$
S_{\phi n}=4 \pi \Delta \nu,
$$

where $\Delta \nu$ is the linewidth of the laser and $\phi_{n}$ is the derivative of the phase noise. The photodiode current with noise is generated and BPF follows. The phase of $i(t)$ after BPF is calculated using inverse tangent and passes through a low-pass filter. The SNR is calculated after the LPF. 


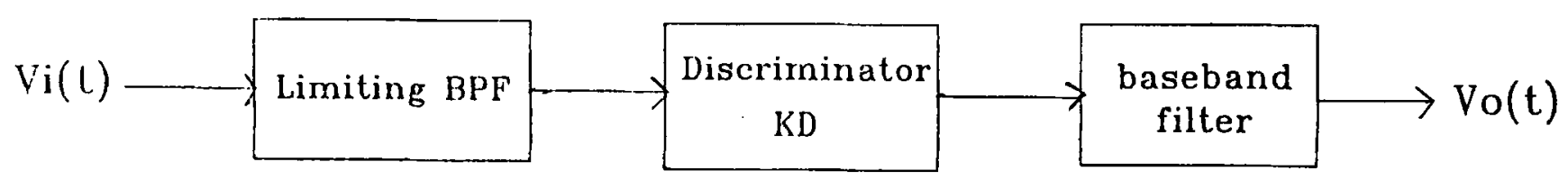

Figure 1 Schematic diagram of the FM receiver

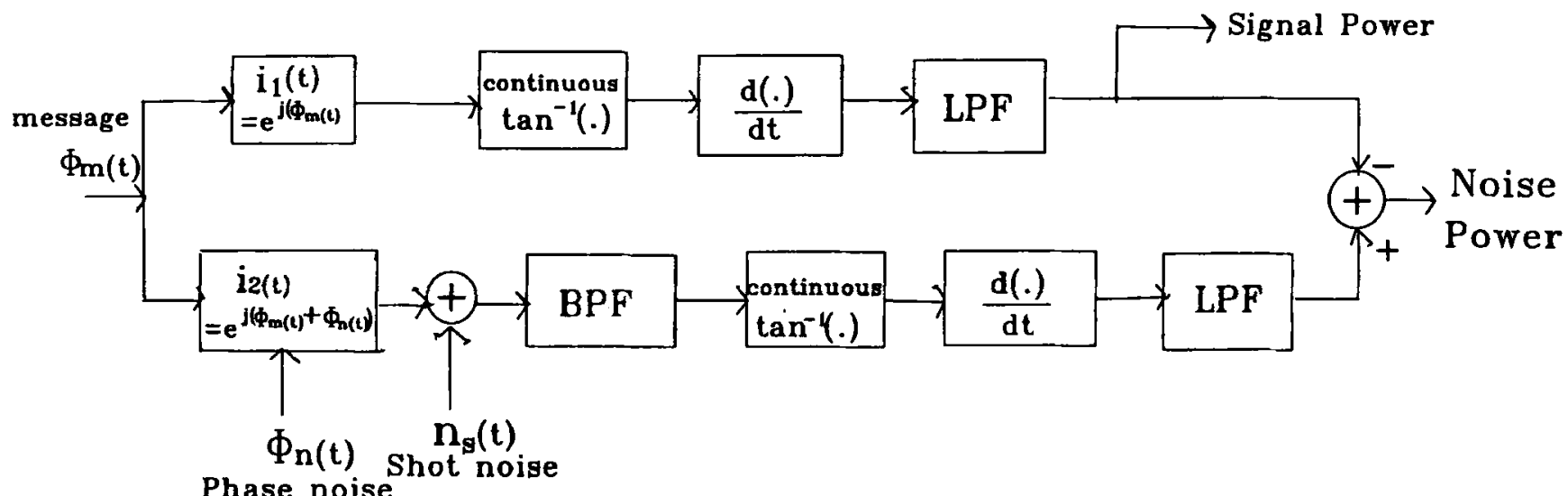

Figure 2 Schematic diagram of our simulation procedure

\section{NUMERICAL RESULTS}

3.1. Simulation of Analog System. In the simulation of analog systems, some factors must be considered in order to get the proper result. They are

1. Sample rate: The sample rate must be sufficiently large to avoid aliasing noise due to the Nyquist sampling theorem [6].

2. The definition of SNR: SNR is defined as the standard derivative of the difference between the output pass through the system ( $B_{\text {i.f. }}=$ sufficiently large) without added noise, and the output pass through the system (specific $B_{\text {i.f. }}$ ) with added noise. Assume $\phi_{m}(t)$ is the FM signal. Then let

$$
\begin{aligned}
i_{1}(t) & =A e^{j \phi_{m}(t)} \\
i_{2}(t) & =A e^{j\left(\phi_{m}(t)+\phi_{n}(t)\right)}+n_{s}(t)
\end{aligned}
$$

where $A=2 \sqrt{P_{\mathrm{LO}} P_{S}}$. We let $i_{1}(t)$ pass through the system with extremely large BPF and then let $i_{2}(t)$ pass through the system with the specific BPF. The two result signals are compared and the standard derivative of the difference is defined as noise.

3.2. Numerical Example. We run the simulation with two difference modulation indices $\beta=5,10$, with the following parameters: Maximum frequency, $f_{m}=6 \mathrm{MHz}$ (for TV channel occupying $6 \mathrm{MHZ}$; responsivity: $0.84 \mathrm{~A} / \mathrm{W}$; laser linewidth: $\Delta \nu=10 \mathrm{KHz}$ (diode pump Nd-YAG lasers [7]). The numerical result with $\beta=5$ is shown in Figure 3 . The low-pass filter bandwidth is the half bandwidth of the i.f.

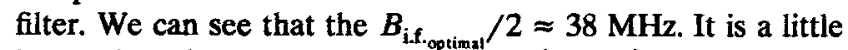
larger than the Carson rule, $B_{\text {i.f. }} / 2=(\beta+1) F_{m}=36 \mathrm{MHz}$, because the effect of phase noise and the Carson rule is an approximation. The i.f. filter can have a large bandwidth if the signal power is larger than $-50 \mathrm{dBm}$ because phase noise

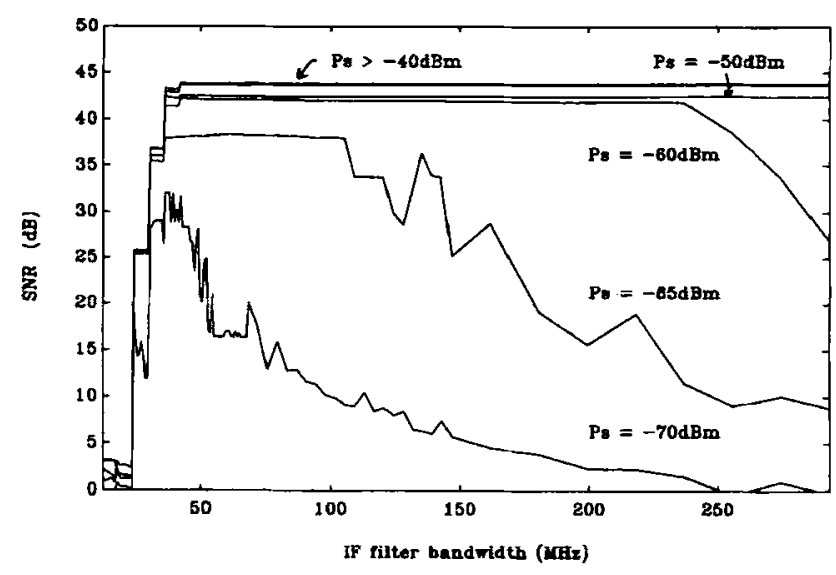

Figure 3 SNR versus difference i.f. filter bandwidth with $\beta=5$

is dominant. SNR will decrease with increasing bandwidth for more shot/thermal noises included.

The numerical result with $\beta=10$ is shown in Figure 4 . The low-pass filter bandwidth is the half bandwidth of i.f.

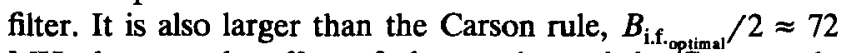
$\mathrm{MHz}$, because the effect of phase noise and the Carson rule is just an approximation. The i.f. filter can have a large bandwidth if signal power is larger than $-50 \mathrm{dBm}$ because phase noise and the shot/thermal noises are dominant. If the signal power is smaller, the SNR will decrease with the increasing bandwidth for more shot/termal noises included. The numerical result with $\beta=10$ is shown in Figure 4 . The low-pass filter bandwidth is the half bandwidth of the i.f. filter. It is also larger than the Carson rule, $B_{\text {i.f. }} / 2=(\beta+$ 1) $f_{m}=36 \mathrm{MHz}$. The effect of signal power on the SNR is similar to $\beta=5$. The SNR curves in both figures have staircases if $B_{\text {i.f. }}<B_{\text {i.f. }}$. Thim is because we do only the single tone simulation. The current, $i(t)$, of the photodiode has a line spectrum, if $B_{\text {i.f. }}$ includes one more line, the SNR 


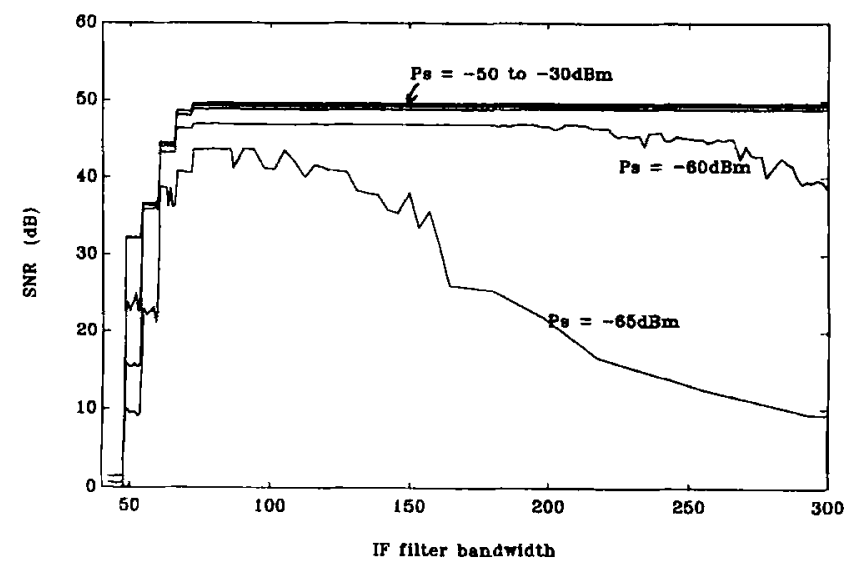

Figure 4 SNR versus difference i.f. filter bandwidth with $\beta=10$

will improve so much. The discontinuities point of the staircase corresponds to $n f_{m}, n>0$. If the message is multitone and distributed more equally, the discontinuous point will disappear. If the signal power becomes smaller, the curve will not be smooth while $B_{\text {i.f. }}>B_{\text {i.f. }}$. We get a smooth curve in that region when the signal power is larger because we use the same series to pass through BPF with different $B_{\text {i.f. }}$ and the domination of phase noise. But simulated shot noise is not white in spectrum and its power spectral density will be larger in one part of the spectrum but smaller in other parts of spectrum. Worse than this, including the noise in one part of the spectrum may cancel the noise in other part of the spectrum. The problem of random number generation dominates the error in simulation [8]. Because we rely on the built-in random number generator of PC - MATLAB ${ }^{\mathrm{TM}}$, we cannot improve the results further.

\section{REFERENCES}

1. Special Issue on Broadband Lightwave Video Transmission IEEE J. Lightwave Technol., Vol. LT-11, No. 1, 1993.

2. H. W. Tsao, J. Wu, and Y. H. Lee, "Performance Analysis of Optical Phase Diversity FSK Receiver Using Delay-and-Multiplying Discriminators," J. Opt. Commun., Vol. 10, No. 3, 1989, pp. $97-100$.

3. R. T. Ziemer and W. H. Tranter, Principle of Communications: Systems, Modulation and Noise (2nd ed.), Houghton Mifflin, Boston, 1985.

4. G. Jacobson and I. Garrett, "The Effect of Crosstalk and Phase Noise in Multichannel Coherent Optical DPSK Systems with Tight IF Filtering," IEEE J. Lightwave Technol, Vol. LT-9, No. 11, 1991, pp. 1609-1617.

5. L. G. Kazovsky, P. Meissner, and E. Patzak, "ASK Multiport Optical Homodyne Receivers," IEEE J. Lightwave Technol., Vol. LT-5, No. 6, June 1987, pp. 770-791.

6. A. V. Oppenheim and R. W. Schafter, Discrete-Time Signal Processing, Prentice-Hall, Englewood Cliffs, NJ, 1989.

7. T. Kane and R. Byer, "Monolithic Unidirectional Single Mode Nd:YAG Ring Laser," Opt. Lett., Vol. 10, 1985, pp. 65-67.

8. M. C. Jeruchim, "Techniques for Estimating the Bit Error Rate in the Simulation of Digital Communication Systems," IEEE J. Selected Areas Commun., Vol. SAC-2, No. 1, Jan. 1984, pp. 153-170.

Received 11-14-94; revised 1-23-95

Microwave and Optical Technology Letters, 9/3, 162-164 (C) 1995 John Wiley \& Sons, Inc.

CCC $0895-2477 / 95$

\section{TRANSMISSION EQUATIONS FOR SOLITON AMPLIFICATION IN ERBIUM-DOPED OPTICAL FIBERS}

\section{Y. Zhao}

Advanced Technology Research Center

Shenzhen University

Shenzhen 518060

People's Republic of China

Department of Electronic Engineering

\section{K. T. Chan}

The Chinese University of Hong Kong, Shatin, N. T., Hong Kong

\section{KEY TERMS}

Optical-fiber amplifiers, soliton, erbium-doped optical fibers

\section{ABSTRACT}

The transmission equation for describing soliton amplification in $\mathrm{Er}^{3+}$ doped optical fibers is derived from the semiclassical theory. To the best of our knowledge, excited-state absorption is considered for the first time in such an equation. The doping shape, pump and signal lightwave modes, and spectral-broadening function are also included. This equation is a general one that is widely applicable to the analysis and optimization of doped fibers for soliton amplification. (c) 1995 John Wiley \& Sons, Inc.

\section{INTRODUCTION}

In optical-fiber soliton communication systems the soliton width becomes broadened and the amplitude gets damped because of the existence of fiber loss. In order to compensate for this loss, soliton amplification is required in high-speed long-haul systems. Although the energy loss of the soliton is the same as that of the general linear pulse [1], the soliton area is constant in propagation. The simple energy compensation can therefore be used to offset the fiber loss. After energy compensation the soliton width will become narrowed and the amplitude will be enlarged until its original shape is recovered.

Hasegawa proposed the use of Raman gain to realize soliton-energy periodic distributed compensation [2], which has since been experimentally confirmed by Mollenauer and Smith [3]. However, this technique requires such a high pump power $(\sim 300 \mathrm{~mW})$ that it is rather difficult to achieve in practical systems. Recently, the technology of optical amplification by $\mathrm{Er}^{3+}$-doped fibers has matured. In comparison with the Raman gain compensation, a much lower pump power (approximately several tens of milliwatts) is needed for the $\mathrm{Er}^{3+}$-doped fiber soliton amplification. Other merits, such as high output saturation power, polarization-independent gain, and near quantum-limited noise also make $\mathrm{Er}^{3+}$. doped fiber the most appropriate soliton amplification medium.

There are two kinds of soliton energy compensation in $\mathrm{Er}^{3+}$-doped fibers. The first is lumped amplification with high doping concentrations, from about $100 \mathrm{ppm}$ to about 1000 ppm in short fibers, the length of which varies from several meters to several hundred meters. The second is distributed amplification with low doping concentrations, from $0.1 \mathrm{ppm}$ to $10 \mathrm{ppm}$ in long fibers whose length extends from several kilometers to several tens of kilometers. Experimental studies of these two kinds of soliton amplification have been reported [4-6]. Theoretical studies have also been presented [7-12]. Here we will present a new model describing soliton 